\section{WHAT IS SHIGELLOSIS?}

Shigellosis is a bacterial disease caused by infection with Shigella bacteria. Shigellosis can affect anyone; however, children, people with poor immune systems, and the elderly are at the greatest risk.

\section{HOW DO YOU CATCH SHIGELLOSIS?}

Shigellosis is passed from person to person by the faecaloral route, by direct or indirect contact with faecal material. This commonly occurs if hands are not washed properly, particularly after going to the toilet or changing nappies, and as a result of sexual contact. Shigella infections may also be acquired from eating food contaminated with the bacteria. Flies can also carry Shigella and can contaminate food.

A person can have Shigella present in their stools for some weeks and remain asymptomatic, and still pass on infection to others.

\section{WHAT ARE THE SYMPTOMS?}

Infection with Shigella usually results in diarrhoea, fever, nausea, vomiting, and stomach cramps. The stool may often have blood or mucus in it. The symptoms begin 1-7 days (usually 1-3 days) after exposure. Symptoms usually last 4-7 days but sometimes longer.

\section{HOW IS SHIGELLOSIS DIAGNOSED?}

Diagnosis of shigellosis requires the isolation of Shigella bacteria from a stool specimen. Your doctor may order this test.

\section{WHAT IS THE TREATMENT FOR SHIGELLOSIS?}

People with mild infections will usually recover without treatment. Drinking increased amounts of fluid is important to avoid dehydration. Young children (particularly infants) are susceptible to dehydration from diarrhoea, and parents should seek medical attention. Antibiotics including ampicillin, trimethoprim-sulfamethoxazole and ciprofloxacin, can be used to treat severe Shigella infections. However, some Shigella have become resistant to antibiotics and using antibiotics to treat mild cases of shigellosis can make the bacteria more resistant in the future. For this reason, usually only severe cases of shigellosis will be treated with antibiotics.

The use of anti-diarrhoeal drugs is not recommended.

\section{HOW IS IT PREVENTED?}

Thorough washing of vegetables and fruit that is eaten raw is recommended.

Thorough handwashing with soap and water is the most important way to avoid contamination and infection. Hands should be washed after:

- going to the toilet;

- changing nappies;

- any exposures to faecal material.

People with shigellosis should avoid work and should not prepare food while they are sick. Sick children, particularly those in nappies, should be kept home from preschool while they have diarrhoea. Children and adults should avoid swimming until diarrhoea has stopped.

People who work as food handlers or who care for children or the elderly should not return to their duties until diarrhoea has stopped and two stool samples-taken at least 24 hours apart and at least two days after any antibiotics have finished-test negative for Shigella.

For further information please contact your local public health unit, community health centre, or doctor.

September-October 2002 \& 